\title{
Effects of a soft-core Coulomb potential on the dynamics of a hydrogen atom near a metal surface
}

\author{
Manuel Iñarrea, ${ }^{1}$ Víctor Lanchares, ${ }^{2}$ Jesús F. Palacián, ${ }^{3}$ \\ Ana I. Pascual, ${ }^{2}$ J. Pablo Salas ${ }^{\text {a }},{ }^{1}$ and Patricia Yanguas ${ }^{3}$ \\ ${ }^{1}$ Área de Física, Universidad de La Rioja, 26006 Logroño, La Rioja, Spain ${ }^{\dagger}$ \\ ${ }^{2}$ Departamento de Matemáticas y Computación, \\ Universidad de La Rioja, 26006 Logroño, La Rioja, Spain \\ ${ }^{3}$ Departamento de Estadística, Informática y \\ Matemáticas and Institute for Advanced Materials, \\ Universidad Pública de Navarra, 31006 Pamplona, Navarra, Spain
}

\begin{abstract}
The goal of this paper is to investigate the effects that the replacement of the Coulomb potential by a soft-core Coulomb potential produces in the classical dynamics of a perturbed Rydberg hydrogen atom. As example, we consider a Rydberg hydrogen atom near a metal surface subjected to a constant electric field in the electron-extraction regime. Thence, the dynamics of the real perturbed Coulomb system, studied by applying the Levi-Civita regularization, is compared with that of the softened one. The results of this study show that the global behavior of the system is significantly altered when the original Coulomb potential part is replaced by a soft-core potential.

Keywords: Coulomb-like potential; soft-core potential approximation; periodic orbits; ionization dynamics.
\end{abstract}

(c) 2018. This manuscript version is made available under the CC-BY-NC-ND 4.0 license http://creativecommons.org/licenses/by-nc-nd/4.0/

\footnotetext{
a Corresponding author

$\dagger$ manuel.inarrea@unirioja.es
} 


\section{INTRODUCTION}

The behavior of Rydberg atoms exposed to external fields is one of the fundamental problems in physics. Except for the hydrogen atom, where the atomic potential is given by a pure Coulomb potential, the interaction between the outer electron and the ionic core in nonhydrogenic Rydberg atoms is frequently described by means of Coulomb-like pseudopotentials. In general, these pseudopotentials are made of a pure Coulomb potential amended with suitable angular-dependent terms (see e.g. [1-5]), whose parameters are fitted to reproduce the field-free energy levels of the atom and thence the corresponding empirical quantum defect. A quite different approach to nonhydrogenic atoms is based on the so-called soft-core Coulomb potentials [6-9]. These potentials are built by introducing a parameter $a$ in the pure Coulomb potential $1 / r$, such that it becomes $1 / \sqrt{r^{2}+a^{2}}$, and where the value of the softening parameter $a$ is adjusted to described the particular atom under investigation [10,11]. Although soft-core Coulomb potentials have been applied to study diverse physical phenomena as inverse bremsstrahlung heating in laser-matter interactions [12] or plasma excitations of a Coulomb gas [13], its most frequent application has been in strong field physics $[14,15]$. Indeed, soft-core Coulomb potentials are used to construct one-dimensional atomic models to study single $[15,16]$ and double [17-21] photoinduced ionization processes. A similar approach to soft-core Coulomb potentials was presented in [22]. In that paper, the ionic core was modeled by adding a suitable term to the Coulomb potential that was appropriately adjusted to reproduce the corresponding quantum defect. Using this modified potential and the semiclassical closed-orbit theory [23-25], the authors were able to reproduce with accuracy the photoabsorption spectra of helium in a magnetic field.

From the point of view of the nonlinear dynamics, several classical studies $[10,11,26]$ using soft-core Coulomb potentials have obtained a remarkable success in the explanation of the experimental behavior of the double ionization probability versus the field intensity of atoms in the presence of linearly or circularly polarized laser fields [27-29]. In particular, Kamor et al. [26] and Mauger et al. [10] showed that the remarkable differences encountered in those experimental results, which depend on the atom used, can be explained by a suitable selection of the value of the softening parameter $a$.

As a result of the success of the combined used of nonlinear mechanics and a soft-core 
Coulomb potential, we address in this paper the study of the possible effects that the application of the later can produce in the global classical dynamics of a hydrogenic system. In other words, we investigate the effects of the soft-core approximation when it is applied beyond one-dimensional atoms. In addition, this investigation is also motivated by the results of Santoprete and Stoica in their study on the phase space flow in smoothed central field problems $1 / r^{\alpha}, \alpha>0$ [30]. Indeed, these authors showed that, outside the collision manifold and for $\alpha<2$ which includes the Coulomb case, the system dynamics given by both the real and the soft-core potentials are equivalent. With these conclusions in mind, in the present work we focus on the study of the effect that a soft-core potential can produce in the global dynamics of a perturbed Coulomb system. In particular, we concentrate on the dynamics of a Rydberg hydrogen atom near a metal surface subjected to a constant electric field in the electron-extraction regime $[31,32]$. Thence, we compare the results obtained in the study of the classical dynamics of this perturbed Rydberg system when the atom is modeled using a pure Coulomb term, to the results when a soft-core approximation for the atomic potential is used. When dealing with the pure Coulomb term, we apply the Levi-Civita regularization technique [33] in order to elude the numerical troubles related to the potential divergence at the origin.

The paper is organized as follows. In Sec. II, the system object of study is described. The main features of the real potential and its soft-core approximation are also provided. The next section is devoted to study, in a comparative way and as a function of the parameters of the system, the dynamics of the Rydberg hydrogen atom using both versions of the potential. To this end, we use Poincaré surfaces of section in the low energy regime. Sec. IV is focussed on the ionization dynamics of the Rydberg atom in the high energy regime. Finally, the conclusions are presented in Sec. V. Atomic units are used throughout the paper.

\section{RYDBERG HYDROGEN ATOM NEAR A METAL SURFACE}

We consider the classical motion of an electron in a Coulomb field created by an infinitely massive nucleus of charge $e>0$ located at the origin of a reference frame $X Y Z$. A plane metal surface perpendicular to the $Z$-axis is located at $z=-d$. Besides, a uniform constant electric field of strength $f$ is applied along the $Z$-axis. As the system has axial symmetry around the $Z$-axis, the use of cylindrical variables $\left(\rho, z, \phi, P_{\rho}, P_{z}, P_{\phi}\right)$ is convenient. The 
Hamiltonian $\mathcal{H}$ (i.e. the energy $E$ ) of the electron expressed in these variables and in atomic units is given by

$$
E \equiv \mathcal{H}=\frac{P_{\rho}^{2}+P_{z}^{2}}{2}+U(\rho, z)
$$

where

$$
U(\rho, z)=\frac{P_{\phi}^{2}}{2 \rho^{2}}-\frac{1}{\sqrt{\rho^{2}+z^{2}+a^{2}}}+f z+\frac{1}{\sqrt{\rho^{2}+(2 d+z)^{2}}}-\frac{1}{4(d+z)}
$$

The second term of the effective potential $U(\rho, z)$ stands for the Coulomb interaction between the electron and the nucleus. For the sake of completeness, in this term we have introduced the soft-core parameter $a$, such that, when $a=0$, we have the real Coulomb potential. When the soft-core model is considered, the typical values for $a$ are in the range $0.8 \leq a \leq 2$ [15]. In particular, we use in this paper the value $a=2$. The last two terms of the potential (2) account for the electric image model describing the interaction of the electron with the metal surface. For more details on the model, we refer the reader to [34].

Due to the axial symmetry of the system, the $z$ component $P_{\phi}$ of the angular momentum is conserved and (1) defines a two-degree-of-freedom dynamical system. Then, the dynamics of the electron depends on four parameters: the energy of the system $E=\mathcal{H}, P_{\phi}$, and the external parameters $d$ and $f$. In this paper, we restrict ourselves to the case $P_{\phi}=0$, so that the number of parameters reduces to three: $E=\mathcal{H}, d$ and $f$. We restrict to the case $P_{\phi}=0$ because for $P_{\phi} \neq 0$ the infinite well of the real effective potential located at the origin no longer exists. Instead of it, the real effective potential presents a finite potential well located outside the origin. Therefore, in the case $P_{\phi} \neq 0$ there is no need to apply any softening to the potential. In this study we are interested in the effects that softening can create on the dynamics of this system. This model has already been studied by the authors applying regularization to the real Coulomb potential, $a=0[31,32]$. 


\section{A. Particular solutions: Rectilinear orbits along the $Z$-axis}

The equations of motion of Hamiltonian (1) for $P_{\phi}=0$ are

$$
\begin{aligned}
\frac{d \rho}{d t} & =P_{\rho}, \quad \frac{d z}{d t}=P_{z}, \\
\frac{d P_{\rho}}{d t} & =\frac{\rho}{\left((2 d+z)^{2}+\rho^{2}\right)^{3 / 2}}-\frac{\rho}{\left(\rho^{2}+z^{2}+a^{2}\right)^{3 / 2}}, \\
\frac{d P_{z}}{d t} & =\frac{2 d+z}{\left((2 d+z)^{2}+\rho^{2}\right)^{3 / 2}}-\frac{1}{4(d+z)^{2}}-f-\frac{z}{\left(\rho^{2}+z^{2}+a^{2}\right)^{3 / 2}},
\end{aligned}
$$

It is straightforward to show that rectilinear orbits along the $Z$-axis are particular solutions of the equations of motion (3). For historical reasons, we name these rectilinear orbits as $I_{z}$ [35]. There is a significant difference in the nature of $I_{z}$ depending upon the system is whether or not softened. Indeed, when $a=0$ it is well known that solutions $I_{z}$ are made of two disjoint families of trajectories along the positive $Z$-axis $\left(I_{z}^{+}\right)$and along the negative $Z$-axis $\left(I_{z}^{-}\right)$, respectively (see e.g.[32]). These two families are kept apart by the Coulomb singularity. However, when $a \neq 0$, the Coulomb singularity is removed and the electron can pass through the origin, such that the solutions $I_{z}$ belong to a unique family of trajectories along the $Z$-axis. As we will see shortly, this difference in the nature of $I_{z}$ will have an important impact on the global dynamics of the system.

\section{B. The critical points of the effective potential $U(\rho, z)$}

When the electric field is directed to the metal surface $(f<0)$, the effective potential $U(\rho, z)$ presents two saddle points in both the real Coulomb potential $(a=0)$ and its softcore approximation $(a \neq 0)$. One of these saddle points is located in the negative part of the $Z$-axis, $S_{-} \equiv(\rho, z)=\left(0, z_{s p}^{-}\right)$with $z_{s p}^{-} \in \mathbb{R}^{-}$, and the other one is located in positive part of the $Z$-axis, $S_{+} \equiv(\rho, z)=\left(0, z_{s p}^{+}\right)$with $z_{s p}^{+} \in \mathbb{R}^{+}$. Theses saddle points are the two possible ionization channels of the atom for high enough values of the energy. The saddle point $S_{-}$is the potential barrier that the electron must overcome to be captured by the metal surface. On the other hand, the saddle point $S_{+}$is the opposite potential barrier through which the electron can be dragged to the vacuum by the applied electric field.

Fig. 1 shows the curves of the effective potential $U(\rho, z)$ along the direction $\rho=0$, for several values of the metal-nucleus distance $d$ and for a fixed electric field strength 
$f=-5 \times 10^{-6}$ a.u. In this figure, the solid lines correspond to the real Coulomb potential $(a=0)$, and the dashed lines stand for the soft-core potential approximation $(a=2)$. As it can be seen in this figure, for each metal-nucleus distance $d$, the curves of both versions of the effective potential do not present significant differences for intermediate and large values of $z$. Nevertheless, these curves start to differ for small values of $\rho$ and $z$. Indeed, Fig. 2 shows the curves of both versions of $U(\rho=0, z)$ in the vicinity of the origin for $f=-5 \times 10^{-6}$ a.u. and $d=300$ a.u. In this figure it is clear that both potential curves are different for $|z| \lesssim 5$ a.u.

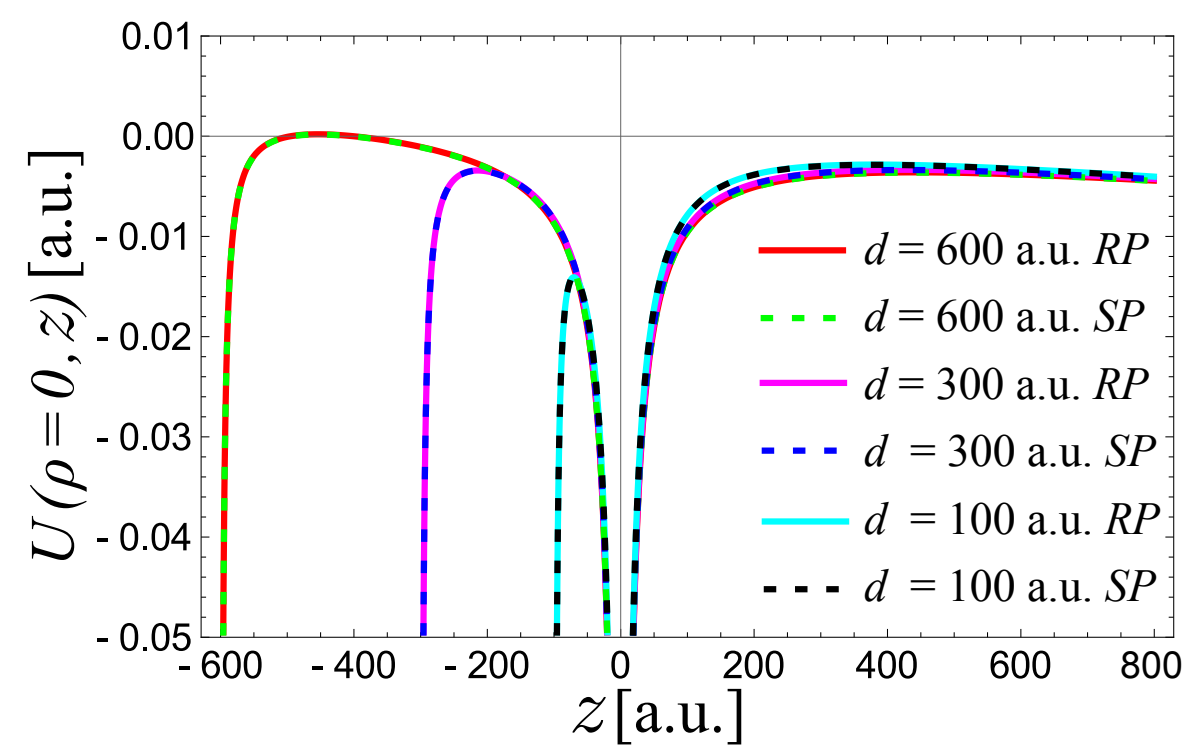

FIG. 1. Effective potential curves $U(\rho=0, z)$ corresponding to both versions of the potential for several values of the metal-nucleus distance $d$. The labels $R P$ and $S P$ stand for real and soft-core potentials, respectively. The electric field strength is fixed $f=-5 \times 10^{-6}$ a.u.

Fig. 1 also shows that, for a fixed value of the electric field strength, the metal surface potential barrier decreases as the metal-nucleus distance $d$ decreases. The opposite occurs with the other potential barrier located at the positive side of the $Z$-axis. This behavior can be seen in Fig. 3 which shows the evolution of the energies of the saddle points as a function of the distance $d$ for both versions of the effective potential. It is worth to note that there is no difference between the evolution of the saddle energies in both versions of the potential. 


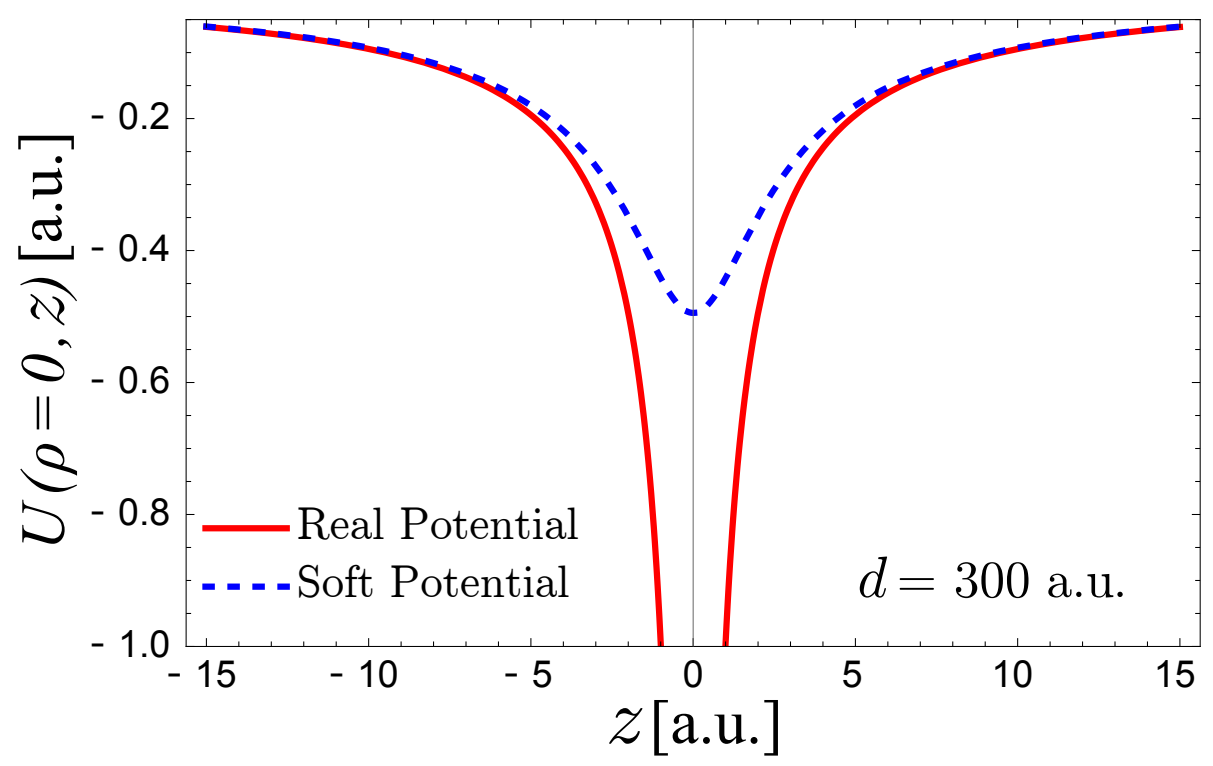

FIG. 2. Effective potential curves $U(\rho=0, z)$ corresponding to both versions of the potential in the vicinity of the origin for $d=300$ a.u. and $f=-5 \times 10^{-6}$ a.u.

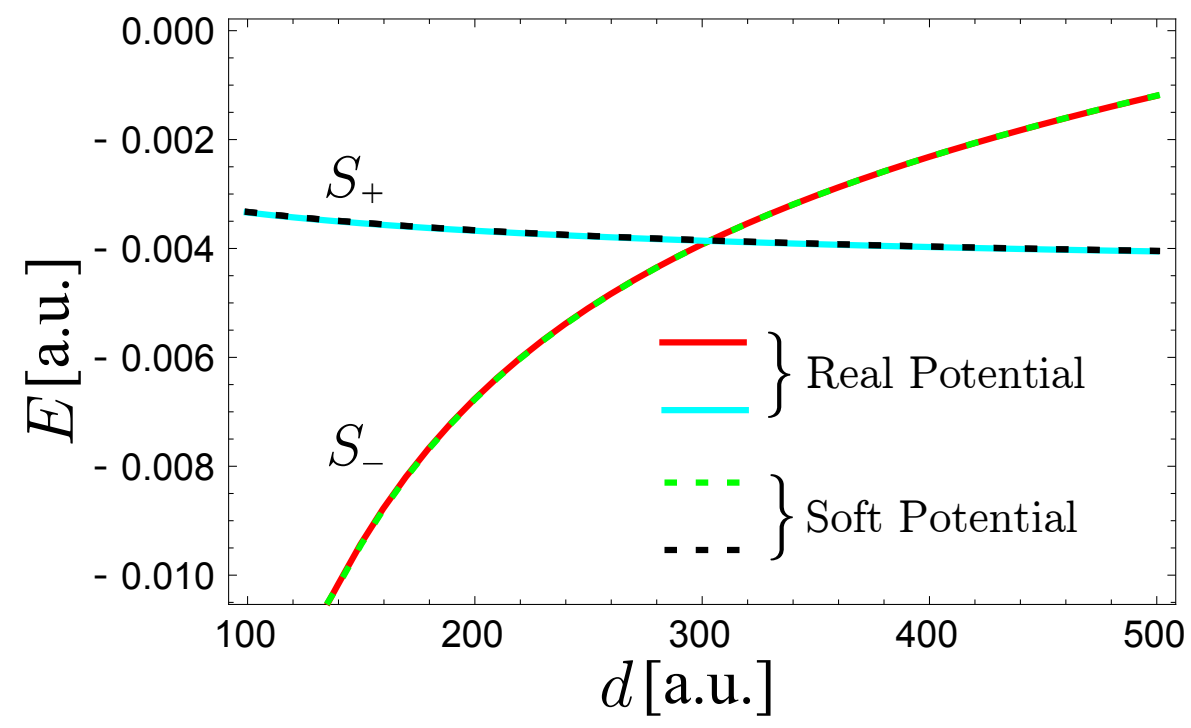

FIG. 3. Evolution of the energies of the saddle points of both versions of the effective potential $U(\rho, z)$ as a function of the distance $d$ for $f=-5 \times 10^{-6}$ a.u.

\section{PHASE SPACE STRUCTURE}

As it is well known, the phase space structure provides most of the information about the dynamics of a Hamiltonian system. In our case, the phase space of the two-degree-offreedom Hamiltonian (1) is four-dimensional, leading to a three-dimensional energy shell, 
such that we can use Poincaré surfaces of section (PSOS) to uncover its phase space structure. Because we are considering the case $P_{\phi}=0$, the motion in Cartesian coordinates is planar and equivalent to that on any plane containing the $Z$-axis. Thence, in order to cover the motion on the whole orbital plane, we allow the coordinate $\rho$ to take negative values. In our problem a convenient plane for the Poincaré map is given by the intersection of the phase space trajectories with the plane $\left(z, P_{z}\right)$ for $\rho=0$ and $P_{\rho} \geq 0$. Thus, the PSOS are limited by the curves

$$
P_{z}= \pm \sqrt{2\left(\frac{1}{4(d+z)}-\frac{1}{2 d+z}+E-f z+\frac{1}{\sqrt{z^{2}+a^{2}}}\right)} .
$$

Note that the rectilinear orbits $I_{z}$ along the $Z$-axis are tangent to the phase flow in this map. For $a=0$, the curves (4) are singular when $|z| \rightarrow 0$, such that, the branch with $z>0$ and the branch with $z<0$ of (4) correspond to the families $I_{z}^{+}$and $I_{z}^{-}$, respectively. For $a \neq 0$, the two branches (4) define a closed curve which corresponds to the (single) family $I_{z}$.

In order to visualize the impact of the softening in the phase space structure of the system, we compute PSOS by fixing the nucleus-metal distance $d=200$ a.u. and the energy $E=-0.02$ a.u., while the electric field value $f$ is used as an external parameter ranging in the interval $-2 \times 10^{-6}$ a.u. $\leq f \leq 0$. When $f=0$ and $d \rightarrow \infty$, this energy corresponds to the energy of an unperturbed hydrogen atom with principal quantum number $n=5$. Because the computation of PSOS requires the numerical integration of phase trajectories, we elude the numerical problems involved with the Coulomb singularity when $a=0$, by applying the so-called Levi-Civita regularization [33]. This procedure consists in a change of coordinates from the cylindrical ones $(\rho, z)$ to a new set of (semiparabolic) coordinates $(u, v)$,

$$
\begin{aligned}
& \rho=u v, \quad z=\left(u^{2}-v^{2}\right) / 2, \\
& u= \pm \sqrt{r+z}, \quad v= \pm \sqrt{r-z} .
\end{aligned}
$$

Then, a new scaled time $\tau$, defined by $d \tau=d t /\left(u^{2}+v^{2}\right)$, is introduced such that, after an overall multiplication by $u^{2}+v^{2}$, Hamiltonian (1) converts to the new Hamiltonian $\mathcal{K}$,

$$
\mathcal{K}=2=\frac{P_{u}^{2}+P_{v}^{2}}{2}-E\left(u^{2}+v^{2}\right)+\frac{2\left(u^{2}+v^{2}\right)}{\sqrt{4 d u^{2} v^{2}+\left(4+u^{2}-v^{2}\right)^{2}}}-\frac{u^{2}+v^{2}}{2\left(2 d+u^{2}-v^{2}\right)}+\frac{f}{2}\left(u^{4}-v^{4}\right),
$$


where

$$
P_{u}=v P_{\rho}+u P_{z}, \quad P_{v}=u P_{\rho}-v P_{z}
$$

Thence, the regularized Hamiltonian (6) takes the constant value 2, the energy $E$ appears as a parameter in $\mathcal{K}$, and the Coulomb singularity is avoided. Furthermore, when only the electric field interaction is considered $(d \rightarrow \infty)$, we clearly observe in Hamiltonian $\mathcal{K}$ the well known integrability behavior of the Stark effect due to its separability in semiparabolic coordinates. Note that this integrability is lost when a soft-core Coulomb potential is used instead of the real one. The regularized equations of motion of Hamiltonian (6) are given by

$$
\begin{array}{cl}
\frac{d u}{d \tau}=P_{u}, & \frac{d v}{d \tau}=P_{v} \\
\frac{d P_{u}}{d \tau}=-\frac{\partial \mathcal{K}}{\partial u}, & \frac{d P_{v}}{d \tau}=-\frac{\partial \mathcal{K}}{\partial v}
\end{array}
$$

For more details about this change of variables, we refer the reader to $[32,33]$. In this way, once the numerical integration in semiparabolic variables $\left(u, P_{u}, v, P_{v}\right)$ of a given trajectory is performed, the trajectory in the original cylindrical coordinates is obtained by applying the inverse transformation. Using the transformations (5), the Poincaré map $\rho=0$ is equivalent to the conditions $u=0$ and/or $v=0$ in regularized coordinates. Then, for the sake of completeness, we will show the surface of section $v=0$ in regularized coordinates when we use the real Coulomb potential. On the other hand, when the soft-core approximation of the Coulomb potential $(a \neq 0)$ is used, the effective potential $U(\rho, z)$ no longer exhibits singular points, so that, cylindrical coordinates are used during the numerical integration.

In Fig. 4 the surfaces of section $\rho=0$ and $v=0$ are depicted in cylindrical and regularized coordinates respectively for the real (left panel) and for the softened (right panel) Coulomb potentials when the electric field is zero. We observe that, in cylindrical coordinates, both panels show the same qualitative structure. Indeed, the branch with $z>0$ and the branch with $z<0$ of the limit (4) of the PSOS of Fig. 4(a) in cylindrical coordinates correspond to the linear orbits $I_{z}^{+}$and $I_{z}^{-}$, respectively. In the regularized Poincaré map $v=0$ of the inset in Fig. 4(a), these rectilinear orbits $I_{z}^{+}$and $I_{z}^{-}$along the positive and the negative $Z$-axis correspond to the limit of that regularized section and to its central stable fixed point, respectively. On the other side, the limit of the section in Fig. 4(b) represents the rectilinear orbit $I_{z}$. The KAM tori around these periodic orbits are filled in with librational 

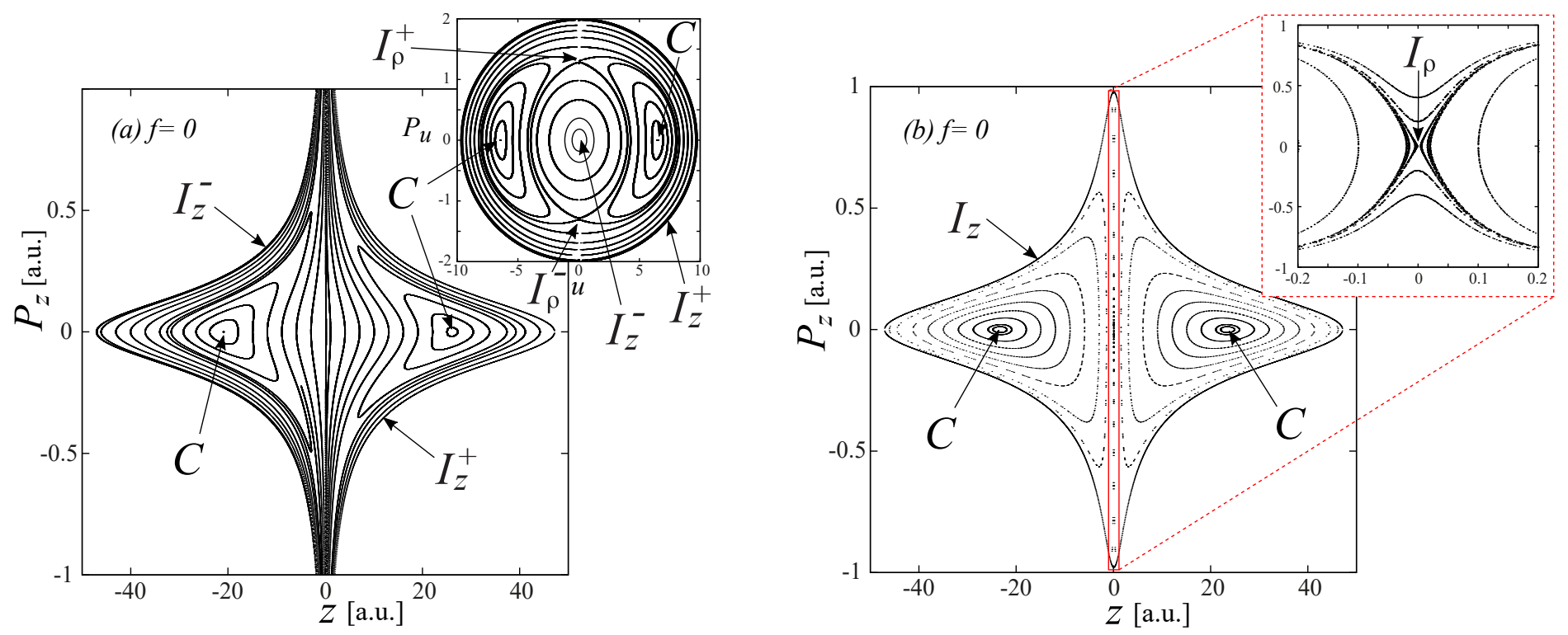

FIG. 4. Poincaré surfaces of section (PSOS) $\rho=0$ for $f=0$ : (a) Real Coulomb potential, (b) soft-core potential $(a=2)$. The inset in the left figure is the Poincaré section $v=0$ in regularized coordinates. All sections are calculated for a nucleus-metal distance $d=200$ a.u. and for an energy $E=-0.02$ a.u.

quasiperiodic orbits mainly localized along the $Z$-axis. Note that these quasiperiodic orbits connect, separately, the core with the distant phase space regions located at the positive and the negative $Z$-axis. Moreover, in both sections in Fig. 4(a)-(b) two stable fixed points appear located at the $P_{z}=0$ axis, e.g. at the $P_{u}=0$ axis in the regularized Poincaré map of the inset of Fig. 4(a). We name these periodic orbits as $C$ and they are almost circular orbits travelled in opposite directions. The invariant tori around these elliptic points (stable periodic orbits) are filled in with rotational quasiperiodic orbits with the same symmetry pattern as $C$. The Poincaré map in regularized coordinates [see the inset in Fig. 4(a)] also shows a separatrix passing through two unstable (hyperbolic) fixed points located at the $u=0$ axis. We name these hyperbolic points as $I_{\rho}^{+}$and $I_{\rho}^{-}$and they correspond to (almost) rectilinear periodic orbits mainly aligned along the positive and the negative $\rho$-axis respectively. These hyperbolic points $I_{\rho}^{ \pm}$as well as the separatrix cannot be observed in the cylindrical representation of the PSOS in Fig. 4(a) because the fixed points $I_{\rho}^{ \pm}$would be located at $z=0$, and when $z \rightarrow 0$ the Poincaré map in cylindrical variables becomes singular. However, when the soft-core potential is used, the origin $(\rho, z)=(0,0)$ is not singular and the periodic orbits $I_{\rho}^{ \pm}$appear as a single hyperbolic fixed point $I_{\rho}$ at the origin 

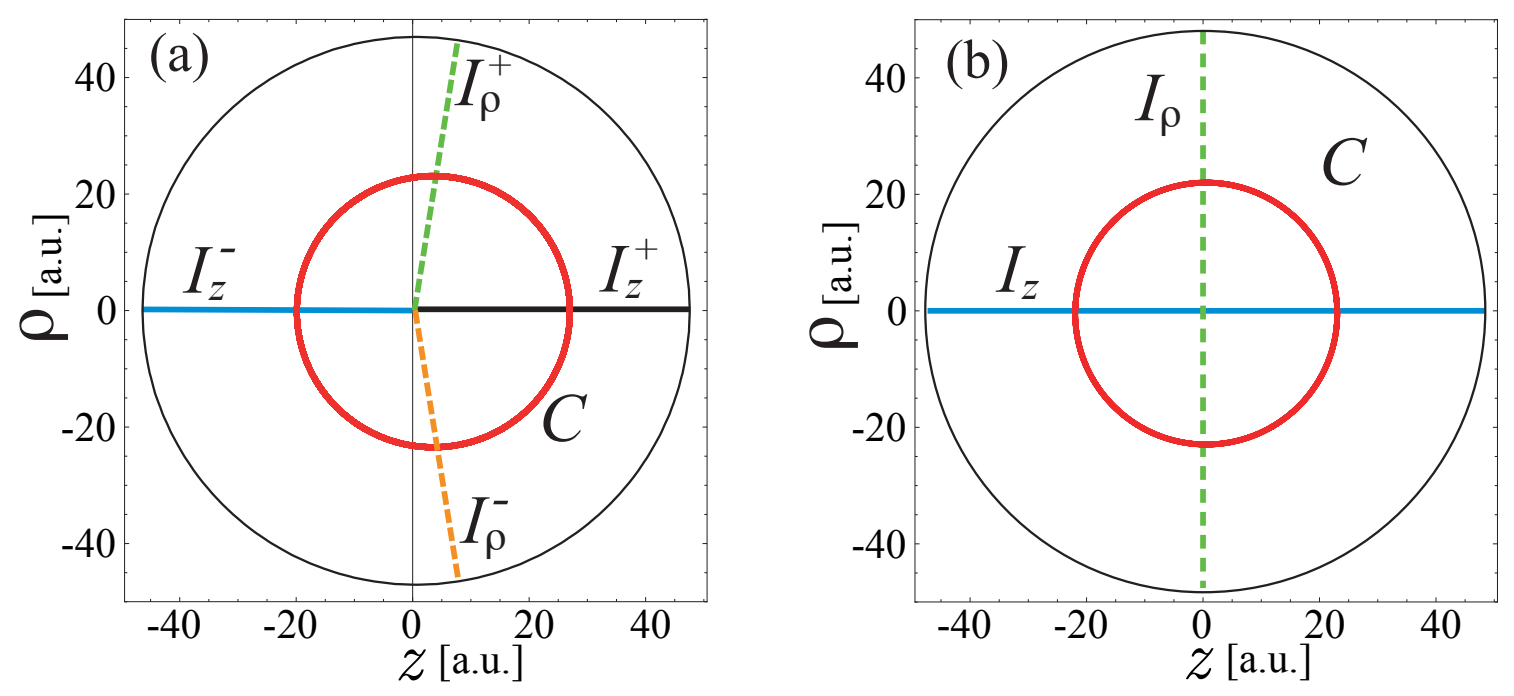

FIG. 5. Periodic orbits: (a) Real Coulomb potential and (b) soft-core potential $(a=2)$. Dashed lines indicate instability. All orbits are calculated for zero electric field, nucleus-metal distance $d=200$ a.u. and energy $E=-0.02$ a.u. The outer black lines correspond to the equipotential curves with energy $E=-0.02$ a.u.

that corresponds to a single periodic orbit along the $\rho$ axis. This unstable structure is observed in the zoom of the inset of Fig. 4(b). All these periodic orbits $I_{z}^{ \pm}, C$ and $I_{\rho}^{ \pm}, I_{z}$ and $I_{\rho}$ are depicted in Fig. 5. It is worth noting that, as a consequence of the softening most of the phase space is filled in with rotational quasiperiodic orbits around the stable periodic orbits $C$ [see Fig. 4(b)]. In other words, the softened potential pushes most of the vibrational orbits around $I_{z}$ out of the phase space. Then, the aforementioned existing connection in the real Coulomb potential between the core and the distant phase space regions along the $Z$-axis is lost.

Now, when the electric field $f$ is turned on, we study its impact on the structure of the phase spaces of Fig. 4. Indeed, in Fig. 6 the Poincaré maps for decreasing values of the electric field $f=-1 \times 10^{-6}$ a.u., $f=-1.2 \times 10^{-6}$ a.u. and $f=-2 \times 10^{-6}$ a.u. are shown in the soft (right column) and in the real cases (left column). When the real Coulomb potential is used, we observe in the surfaces of section $\rho=0$ of the left column of Fig. 6 that, for decreasing field values, the elliptic fixed point $C$ located at the negative part of the $Z$-axis moves towards the limit of the surface, while the fixed point $C$ at the positive part of the $Z$-axis tends to the origin. This behavior becomes clear in the surfaces of section in regularized coordinates of Fig. 6(a), where a migration of the elliptic fixed 
points $C$ towards the central fixed point of the surface of section, e.g. the fixed point $I_{z}^{-}$, is observed. Moreover, in the regularized sections we observe that the hyperbolic fixed points $I_{\rho}^{ \pm}$tend also to the central point $I_{z}^{-}$. As a consequence, the regions of KAM tori around the periodic orbits corresponding to the fixed points $C$ shrink. Then, the former shape of the periodic orbits associated to $C$ and $I_{\rho}^{ \pm}$for $f=0$ [see Fig. 5] is lost and, as we can observe in Fig. 7 for $f=-1 \times 10^{-6}$ a.u., they gradually adapt to the shape of $I_{z}^{-}$. For decreasing values of the field strength, the migration of the families $I_{\rho}^{ \pm}$and $C$ towards $I_{z}^{-}$continues, such that at $f \approx-1.202 \times 10^{-6}$ a.u. a subcritical pitchfork bifurcation occurs when the unstable families $I_{\rho}^{ \pm}$and the stable periodic orbit $I_{z}^{-}$collide. From this collision, only $I_{z}^{-}$, which becomes unstable, survives [see Fig. 6(c)]. At $f \approx-1.578 \times 10^{-6}$ a.u. a supercritical pitchfork bifurcation takes place when the stable families $C$ and the (unstable) $I_{z}^{-}$orbit come into coincidence. From this second bifurcation only $I_{z}^{-}$survives, becoming stable, see Fig. 6(e). After passing this bifurcation, the rotational orbits disappear, and the phase space is made of librational orbits around $I_{z}^{-}$and $I_{z}^{+}$, such that the nearer the librational orbit is to $I_{z}^{-}\left(I_{z}^{+}\right)$, the greater its orientation along the negative (positive) $Z$-axis is. Roughly speaking, the atom is polarized by the electric field because most of the orbits are aligned along the $Z$ direction.

However, the presence of the electric field has a very different impact in the smooth system. As we observe in the Poincaré maps of the right column of Fig. 6, the phase space structure of the softened system is only slightly distorted by the field, such that it remains almost unchanged as the electric field decreases. In other words, none of the two bifurcations described in the real system takes place, which results in that the softening prevents the atom to be polarized by the field. The reason why the field is not able to polarize the atom is not completely clear. It may be related to the fact that softening prevents the existence of quasiperiodic orbits around the periodic orbit $I_{z}$ for $f=0$. So that, for $f \neq 0$, the quasiperiodic orbits around $C$ that fill most of the phase space can not be polarized by the electric field. Roughly speaking, there is no place in the phase space for quasiperiodic motions oriented along the $Z$-axis.

So far, we have studied the evolution of the phase space for fixed metal-nucleus distance $d=200$ a.u. and decreasing values of the electric field strength. Now, we analyze the effect of the metal-nucleus distance $d$ on the structure of the phase space for a fixed value of the electric field strength $f=-5 \times 10^{-6}$ a.u. The results of this study for decreasing distances 

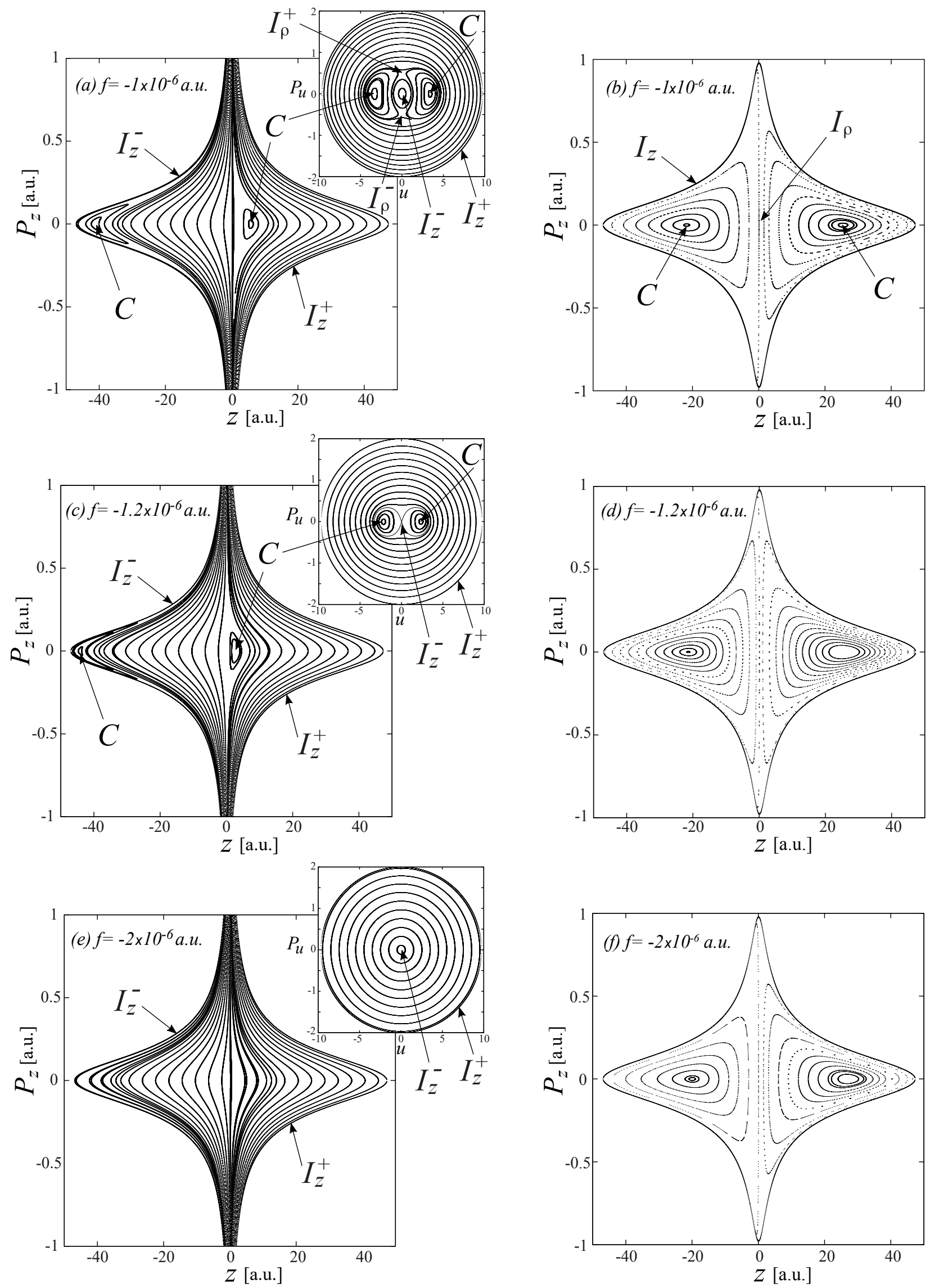

FIG. 6. Evolution of the Poincaré surfaces of sefţion (PSOS) $\rho=0$ for: (a)-(b) $f=-1 \times 10^{-6}$ a.u., (c)-(d) $f=-1.2 \times 10^{-6}$ a.u. and (e)-(f) $f=-2 \times 10^{-6}$ a.u. Left column: real Coulomb potential. Right column: soft-core potential $(a=2)$. All sections are calculated for a metal-nucleus distance $d=200$ a.u. and energy $E=-0.02$ a.u. 


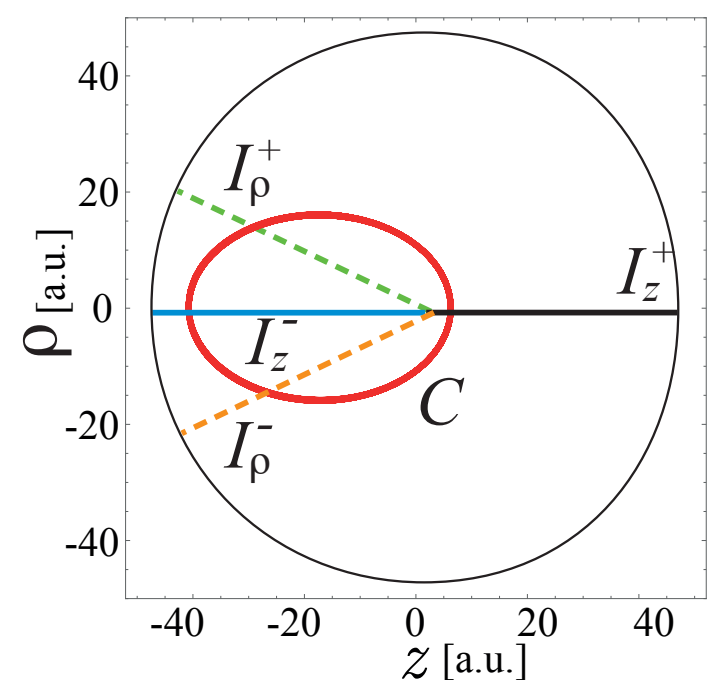

FIG. 7. Periodic orbits for the real Coulomb potential. Dashed lines indicate instability. All orbits are calculated for the electric field value $f=-1 \times 10^{-6}$ a.u., a nucleus-metal distance $d=200$ a.u. and energy $E=-0.02$ a.u. The outer black curve corresponds to the equipotential curve with energy $E=-0.02$ a.u.

$d=200$ a.u., $d=125$ a.u. and $d=75$ a.u. are shown in Fig. 8. In the real Coulomb potential case, the Poincaré maps depicted in the left column of Fig. 8 indicate that the phase space shows an inverse evolution of that appearing in the left column of Fig. 6. In particular, the aforementioned two pitchfork bifurcations occur in the reverse order between the situations shown in Fig. 8(a) and Fig. 8(c). In this way, decreasing the metal-nucleus distance $d$ with a fixed value of the field strength $f$ is equivalent to increasing $f$ while keeping fixed the distance $d$. This equivalent behavior is due to the scaling property that enjoys Hamiltonian (1) for $a=0$. Indeed, in the real Coulomb potential case, it is possible to scale the cylindrical variables $\mathbf{r}=(\rho, z)$ and $\mathbf{P}=\left(P_{\rho}, P_{z}\right)$ in the form

$$
\mathbf{r}^{\prime}=\mathbf{r} / d, \quad \mathbf{P}^{\prime}=\mathbf{P} d^{2}
$$

such that the dynamics of the system only depends on the scaled energy $E^{\prime}=E d$ and on the scaled electric field $F=f d^{2}$ [31]. On the other hand, in the soft-core potential case, for $f=-5 \times 10^{-6}$ a.u., when the metal-nucleus distance $d$ decreases, the almost circular stable periodic orbits $C$ centered in the origin, suffer a supercritical pitchfork bifurcation. From this bifurcation, two new families of stable periodic orbits, named as $C_{R}$ and $C_{L}$, are born from $C$, which becomes unstable. In Fig. 9 the new families of orbits $C_{R}$ and $C_{L}$ are depicted 
in the $\rho-Z$ plane. As we can observe, $C_{R}$ and $C_{L}$ are almost circular periodic orbits not centered at the origin. It is worth to notice that, in the soft-core potential case for $d=75$ a.u., the surface of section of Fig. 8(f) shows a large region of chaotic dynamics. However, in the real Coulomb potential case, the corresponding Poincaré map of Fig. 8(e) shows regular behavior, as it is can be clearly seen in the Poincaré map in regularized variables.

We conclude this section highlighting that, although the potential is only distorted in the neighborhood of the origin, the introduction of the softening parameter has a great impact in the global dynamics of the system.

\section{DYNAMICS IN THE IONIZATION REGIME}

As the atom ionization is the most relevant dynamical phenomenon that may occur in the high energy regime, in this section we focus on the dynamics of the electron when its energy is high enough to escape from the nucleus' attraction. It has been pointed out in Sec. II that, for negative values of the electric field $(f<0)$, the effective potential $U(\rho, z)$ presents two saddle points located in the $Z$-axis at both sides of the nucleus. Therefore, for high enough energy values, the ionization of the atom can occur through two opposite channels. Thence, the electron can be either captured by the metal surface through the channel corresponding to the negative saddle point, or pulled out by the electric field to the vacuum through the channel located at the positive saddle point.

Our goal is to study the structure and evolution of the ionization basins corresponding to each escape channel as a function of the nucleus-metal distance $d$ for both versions of the effective potential. Then by the numerical integration of the equations of motion, we have calculated the electron trajectories corresponding to initial conditions included in the phase space plane $\rho=0$ with $P_{\rho} \geq 0$. This subspace is the same used to compute the Poincaré surfaces of section studied in the previous section. All the trajectories considered in this study have been calculated for a constant energy $E=-3.472 \times 10^{-3}$ a.u. which corresponds to a principal quantum number $n=12$, for a fixed value of the electric field strength $f=-5 \times 10^{-6}$ a.u., and for increasing values of the nucleus-metal distance $d$.

The color maps in Fig. 10 show, in a comparative way, the evolution of the ionization basins in the $\rho=0$ planar subspace $\left(z, P_{z}\right)$ for both cases: the real Coulomb potential and the soft-core potential approximation $(a=2)$. The number of trajectories computed in every 

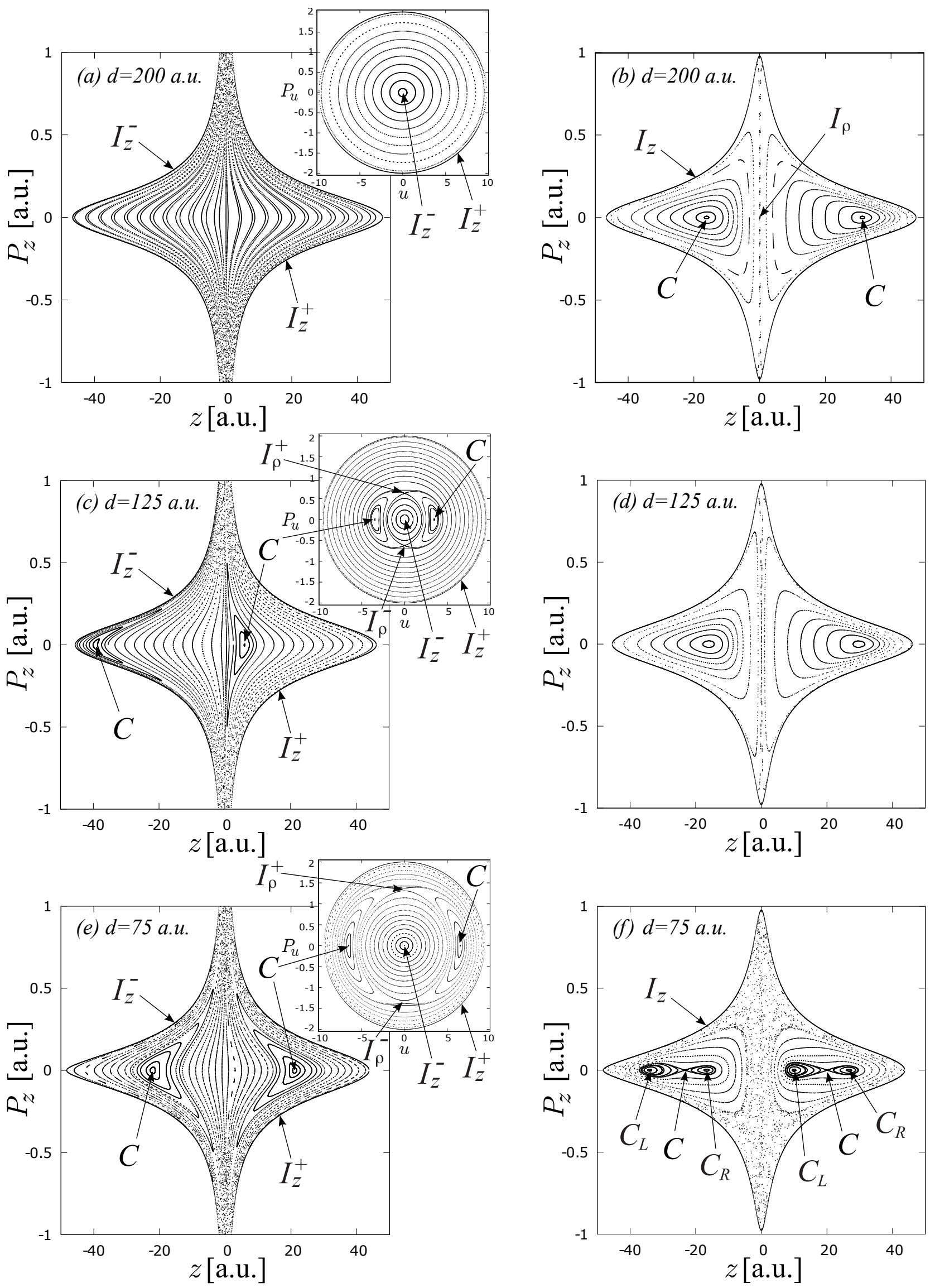

FIG. 8. Evolution of the Poincaré surfaces ofißection (PSOS) $\rho=0$ for: (a)-(b) $d=200$ a.u., (c)-(d) $d=125$ a.u. and (e)-(f) $d=75$ a.u. Left column: real Coulomb potential. Right column: soft-core potential $(a=2)$. All sections are calculated for an electric field $f=-5 \times 10^{-6}$ a.u. and energy $E=-0.02$ a.u. 


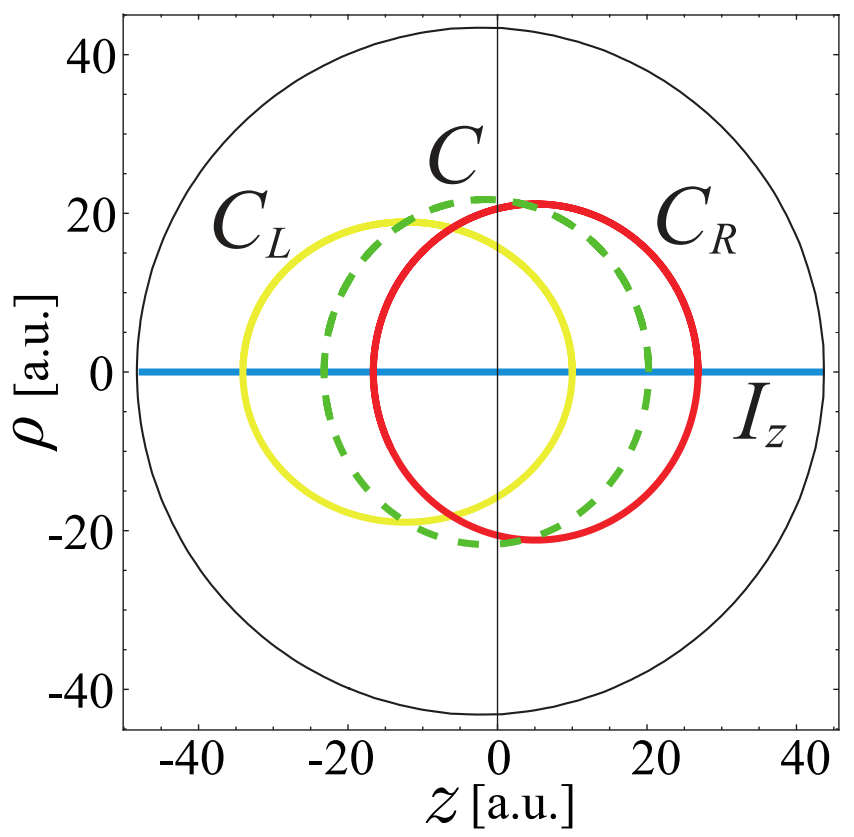

FIG. 9. Periodic orbits in the soft-core potential $(a=2)$, for the electric field value $f=-5 \times 10^{-6}$ a.u., metal-nucleus distance distance $d=75$ a.u. and energy $E=-0.02$ a.u. Dashed line indicates instability. The black line corresponds to the equipotential curve with energy $E=-0.02$ a.u.

plot is around 15000. The cut-off integration time used in these calculations is $t_{f}=10^{5}$ a.u. Red color stands for those initial conditions that lead to the atom ionization through the negative channel, that is, when the electron is captured by the metal surface. Green color represents the initial conditions of ionization trajectories through the positive channel, i.e., when the electron is dragged to the vacuum by the electric field. Blue color accounts for non-ionization initial conditions. In this way, as the metal-nucleus distance $d$ increases, the ionization dynamics evolves through three different regimes. For short distances up to $d \approx 140$ a.u., the influence of the metal surface dominates over the electric field, so that only the negative channel is accessible for the electron to escape towards the metal [see Figs. 10(a)-(b)]. On the contrary, for large values of the surface-atom distance, $d>330$ a.u., the effect of the applied electric field is predominant, and only the positive ionization channel is open [see Figs. 10(g)-(h)]. Finally, for intermediate values of the distance $d$, both channels are accessible for the electron [see Figs. 10(c)-(f)].

As we observe in the color maps of Fig. 10, the ionization features of the electron are different depending on whether the real (left column of Fig. 10) or the soft-core Coulomb potential (right column of Fig. 10) is used. In the former case, the negative and the positive 
ionization basins as well as the non-ionization basin, are separated by smooth curves, without any mixing among them. On the contrary, in the soft-core potential case, there are large regions of initial conditions where the three different basins are strongly mixed, without well defined limits among them.

When the soft-core potential is considered, up to the cut-off time $t_{f}=10^{5}$ a.u. and for any distance $d$, it is always possible to find initial conditions leading to trapped orbits. Nevertheless, it is important to point out that computations performed with different cut-off integration times $t_{f}$ have shown that the number of those non-ionization initial conditions decreases as the $t_{f}$ increases. This decrease of the non-ionization initial conditions with the $t_{f}$ does not happen for the real Coulomb potential. In fact, there always exist sets of initial conditions with a persistent non-ionization behavior. We think this disagreement is due to the different dynamical connection between the core and distant phase space regions for either the real potential and the softened one. On one hand, in the soft-core potential case, most of the phase space is filled in with quasiperiodic orbits around $C$, so that all initial conditions lead to trajectories that can access to any point of the phase space and therefore, they can reach either of the ionization channels. On the other hand, for the real Coulomb potential and for energy values above the energy of the saddle points, the quasiperiodic orbits around $I_{z}^{ \pm}$oriented along the $Z$-axis lead to ionization, whereas some KAM tori persist in the phase space because they are filled in with quasiperiodic orbits around $C$ that are not able to reach none of the ionization channels. Note that in the Poincaré surfaces of section corresponding to the real Coulomb potential appearing in Fig. 6(b) of Ref. [32] there are many orbits that persist. Nevertheless, the Poincaré surfaces of section corresponding to the soft-core potential would appear mostly empty because all trajectories would eventually escape.

\section{CONCLUSIONS}

In this paper we have studied the effects that the application of a soft-core potential produces in the dynamics of a perturbed Coulomb atomic system. In particular, we have taken a Rydberg hydrogen atom near a metallic surface subjected to a constant electric field in the electron-extraction regime. After stating the Hamiltonian of the problem, we have analyzed the particular solutions of the system, as well as the critical points of the effective 

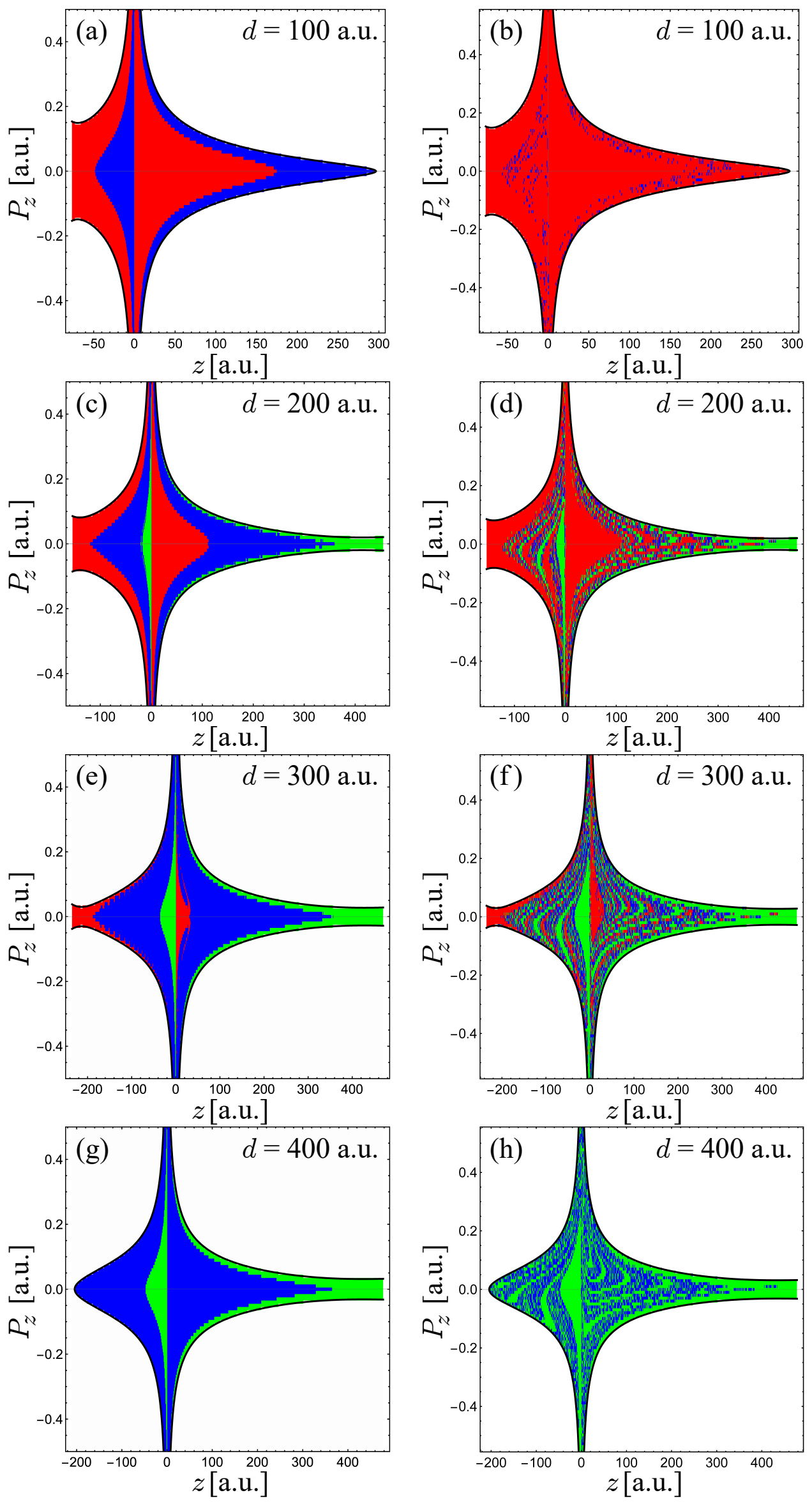

19

FIG. 10. Evolution of the ionization basins in the $\rho=0$ planar subspace $\left(z, P_{z}\right)$ for $f=-5 \times 10^{-6}$ a.u., $E=-3.472 \times 10^{-3}$ a.u. and increasing distances $d$. Left column: real Coulomb potential. Right column: soft-core potential $(a=2)$. Red color: ionization towards the metal surface. Green color: ionization towards the vacuum. Blue color: no ionization. 
potential. From this study we have found that, in the real Coulomb potential, the rectilinear solutions along the $Z$-axis are made of two families, $I_{z}^{+}$and $I_{z}^{-}$, that are kept apart by the Coulomb singularity. However, for the soft-core potential, the electron can pass through the origin and the $I_{z}$ solutions belong to a unique family. This difference in the nature of the rectilinear $Z$ solutions has a crucial effect in the global behavior of the system.

In order to analyze the impact of the softening in the dynamics, we have studied the phase space structure of both, the real and the softened systems, by means of Poincaré surfaces of section. When the energy is fixed well below the ionization channels and when the metalnucleus distance $d$ or the electric field strength $f$ is varied, we have found bifurcations of periodic solutions that appear in the real system and not in the softened one, and viceversa. Moreover, the onset of chaos is made evident in the soft-core Hamiltonian, while the dynamics in the real potential remains regular.

The softening also has a deep influence in the ionization dynamics. Considering the real potential the ionization basins are well defined and there are initial conditions that lead to trajectories that never ionize. However, for the softened potential all initial conditions lead to escape and the ionization and non-ionization regimes are not clearly separated.

We conclude by saying that, although soft-core Coulomb potentials play a relevant role in the study of different processes in strong field physics, the results of this paper indicate that the global dynamics of the perturbed Rydberg hydrogen atom considered in this study is strongly modified when the Coulomb potential is replaced by a soft-core Coulomb potential. In this sense, it is expected that discrepancies will also be found when other perturbations are considered.

\section{ACKNOWLEDGEMENTS}

This work has been partially supported by MTM 2014-59433-C2 of the Ministry of Economy and Competitiveness of Spain and MTM 2017-88137-C2 of the Ministry of Economy, Industry and Competitiveness of Spain.

[1] J. N. Bardsley in Case Studies in Atomic Physics IV, eds. E. W. McDaniel and M. R. C. McDowell (North-Holland Publ. Co., 1974), 299. 
[2] J. Gao and J. B.Delos, Phys Rev A 46, 1455 (1992).

[3] P. A. Dando, T. S. Monteiro, W. Jans, W. Schweizer, Progress of Theoretical Physics Supplement 16, 403 (1994).

[4] C. Oubre, P. Nordlander, and F. B. Dunning. J Phys Chem B 106, 8338 (2002).

[5] F.B. Dunning, H.R. Dunham, C. Oubre and P. Nordlander. Nuclear Instruments and Methods in Physics Research B 203, 69 (2003).

[6] C.H. Mehta and S.H. Patil, Phys Rev A 17, 43-46 (1978).

[7] D. Singh, Y.P. Varshni and R. Dutt, Phys Rev A 32, 619-622 (1985).

[8] F. Grasselli, Am J Phys 85, 834-839 (2017).

[9] D.H. Gebremedhin and C.A. Weatherford, Phys Rev E 89, 053319 (2014).

[10] A. Kamor, F. Mauger, C. Chandre and T. Uzer, Phys Rev Lett 110, 253002 (2013).

[11] F. Mauger, A. Kamor, C. Chandre, T. Uzer, Proceedings of UVX 2012 (June 12-15 2012, Biarritz, France).

[12] R.R. Pandit, Y. Sentoku, V.R. Becker et al, Phys Plasmas 24, 073303 (2017).

[13] F. Grasselli, A. Bertoni and G. Goldoni, Phys Scr 92, 095802 (2017).

[14] P. Agostini and L. Dimauro, Contemp Phys 49, 179 (2008).

[15] W. Becker, X.J. Liu, P.J. Ho and J.H. Eberly, Rev Mod Phys 84, 1011-1043 (2012).

[16] J. Javanainen, J.H. Eberly and Q. Su, Phys Rev A 38, 3430-3446 (1988).

[17] D. Bauer, Phys Rev A 56, 3028-3039 (1997).

[18] R. Panfili, S.L. Haan and J.H. Eberly, Phys Rev Lett 89, 113001 (2002).

[19] R. Panfili, J.H. Eberly and S.L. Haan, Optics Express, 8, 431 (2001).

[20] S.L. Haan, L. Breen, A. Karim and J.H. Eberly, Optics Express, 15, 767-778 (2007).

[21] S.L. Haan, J.S. Van Dyke and Z.S. Smith, Phys Rev Lett 101, 113001 (2008).

[22] B. Hüpper, J. Main, and G. Wunner, Phys Rev Lett 74, 2650 (1995).

[23] M. L. Du and J.B. Delos, Phys. Rev. A 38, 1896 (1988).

[24] M. L. Du and J.B. Delos, Phys. Rev. A 38, 1913 (1988).

[25] J. Main, G. Wiebusch, K. H. Welge, J. Shaw, and J. B. Delos, Phys. Rev. A 49, 847 (1994).

[26] F. Mauger, C. Chandre, and T. Uzer, Phys Rev Lett 105, 083002 (2010).

[27] D. N. Fittinghoff, P. R. Bolton, B. Chang, and K. C. Kulander, Phys Rev A 49, 2174 (1994).

[28] C. Guo and G. N. Gibson, Phys Rev A 63, 040701(R) (2001).

[29] G. D. Gillen, M. A.Walker, and L. D. VanWoerkom, Phys Rev A 64, 043413 (2001). 
[30] M. Santoprete, C. Stoica, Nonlinear Anal Real World Appl 10, 1870 (2009).

[31] M. Iñarrea, V. Lanchares, J.F. Palacián, A.I. Pascual, J.P. Salas and P. Yanguas, Phys Rev A 76, 052903 (2007).

[32] M. Iñarrea, V. Lanchares, J.F. Palacián, A.I. Pascual, J.P. Salas and P. Yanguas, Phys Lett A 379, 131-136 (2015).

[33] T. Levi-Civita, Sur la resolution qualitative du problème des trois corps (University of Bologna Press, Bologna, 1956), Vol. 2. E.L. Stiefel and G. Scheifele, Linear and Regular Celestial Mechanics (Springer-Verlag, Berlin, 1971).

[34] K. Ganesan and K.T. Taylor, J Phys B: At Mol Phys 29, 1293-1306 (1996).

[35] H. Friedrich and D. Wintgen, Phys Rep 183, 37-79 (1989). 